\title{
An Overview of Classification, Requirements, Path Planning Algorithms and Improvement Areas of Unmanned Aerial Vehicle
}

\author{
Princi Sharma \\ CSE, FET \\ MRIIRS, \\ Faridabad
}

\author{
Shivani Singh \\ CSE, FET \\ MRIIRS, \\ Faridabad
}

\author{
Veena Tayal \\ CSE, FET \\ MRIIRS, \\ Faridabad
}

\begin{abstract}
The need of Unmanned Aerial Systems (UASs) is expanding day by day as it can be used in both public and military environments. As the need for UAV is growing, there is an expansion inthe requirement for more reliable, authentic, efficient, optimized and strong vehicles that are capable for executing various operations. The need of such systems is mainly by the Militaries that continue to desire more UAV functionalities for diverse operations and tasks that can be performed all over the world. To have a continuous advancement in the field of autonomous UAV control system many cogent research works has been performed. A large amount of work is focused on the subsets of UAS control such as path planning algorithms, control of small UAV and autonomy. As various markets are amplifying, the necessity to have such systems with capability to adapt according to introduced tasks, sensing elements, and surroundings will drive requirements. They can be used in several ways in various models, sizes and types according to the needs of various operations. The most common Unmanned Aerial Vehicle is "drone"that can be operated by remote controllers using radio waves. These UAVs normally contains internal memory and uses battery power as a means to an energy source. In the current scenario, commercial drones are normally subjected to manual control by human that can control only one drone at a point of time. In accordance to set up a controlling system of multiple drones, collision avoidance mechanism and the detection of objects around these UAV systems is very necessary. This paper discusses about the UAVs requirements and capabilities along with path planning algorithms. It also provides some problems associated with a UAV system along with its improvement areas.
\end{abstract}

\section{Keywords}

Unmanned Aerial System (UAS),autonomous systems, Unmanned Aerial Vehicle(UAV), Drone, path planning algorithms.

\section{INTRODUCTION}

The Unmanned Aerial Vehicle (UAV) is an aircraft that has no pilot in it. Remote controllers can be used to control the UAVs. The UAVs can autonomously be flown. These systems are based on already programmed flight planning paths or more complex aggressive automation applications. A lot of expansion has been done in the field of UAVs. Basically Federal Aviation Administration (FAA) has accepted the Unmanned Aircraft System (UAS) cipher for following the actuality about these aggressive systems also includes other elements and ground stations except the actual air vehicles. The term UAS, however, is not used widely as the term UAV has become more prevalent in the modern vocabulary.
Another recognised type of Unmanned Aerial Vehicle is a Drone that is used in the situations where access of human is not possible[1]. Till today, as the prices are getting cheaper and the drones have become more common and are usually being used for the purpose of entertainment are available easily in the market. Remote controllers through radio waves are used to control these drones[2]. Drones used for commercial purposes aremanually controlled and only a single drone is operated at a time.

The Teal group, in July 2014, forecasted that Unmanned Aerial Systems (UASs) outlay will grow exponentially worldwide. As the growth for such systems continues to grow, difficulties and demands will continue to grow as the customers will have expectations of more efficient, reliable and adapted vehicles. In the defence market the needs have continue to expand, as these markets are expanding day by day, the requirement of systems that can be used in multiple areas such as new operations, sensors, and which can adapt to new environments [3]. UAS technology is known to be the best for the military and applications used for intelligence like targeted assassinations, investigations andaerial supervision[4,5], data and communication interfaces [6]. A lot of civilians are also using these drones and their applications have emerged. Various applications in the field of agriculture, fire fighting, police, sciences, and forestry [7]. Drones have also started to excel in various civilian areas, some of them are 3-D mapping,Hurricane Hunting, down on the farm,protecting wildlife, search and rescue and many more. This paper will constitute the system requirements, problems and improvement areas that are necessary to create a reliable and adaptive system that satisfy to existing and future requirements. In section 2 , we have provided Literature Review. In section 3, we have provided the system requirements that UAV needs to satisfy. In section 4 , we have provided a brief review of the path planning algorithms and safety controls. In section 5, we are discussing various applications of drones in various areas. In section 6, we are discussing various problems in UAS. In section 7 , we are providing some recommended improvement areas. In section 8 , final conclusion is provided.

\section{LITERATURE REVIEW}

In paper [2], the author presents a control system for multiple drones which consists of automatic collision avoidance property. This system uses detection device composed of embedded control device and ultrasonic sensors. The author has also designed a collision avoidance algorithm and claims that the work accuracy of the whole system is more that $90 \%$. In [3], the paper discusses about the system architecture which can be modified depending upon the requirements of the mission and the platform. The paper also discusses about employment of UAS capabilities and current research. In 
paper [8], the authors discusses about the issues present in the autonomous intelligent control of UAVs and the four-layer hierarchal control architecture. In paper [9], the author discusses about the Genetic Algorithm (GA) used to construct flyable trajectory for multiple UAV system. The purposed system is written in the Java language with a Graphical User Interface (GUI) to display the results. In paper [10], the author explains Simulated Annealing (SA) Algorithm used for optimizing the 2D path for UAVs. The paper discusses about the easy threat avoidance methods and applies it to the clarification using SA to bypass from the legitimate circular radar hazard. In paper [11], a new algorithm has been proposed by the author known as Cooperative and Geometric Learning (CGL) to clarify the problems while occurring in planning path for Unmanned Aerial Vehicles (UAVs). The authors also discusses about the contribution of CGL.

Table1. Brief Explanation of Application

\begin{tabular}{|c|c|c|c|c|}
\hline S no. & Year & Author & Application & Explanation \\
\hline 1. & 2015 & K.Surakul & $\begin{array}{l}\text { An application using Automatic } \\
\text { Collision Avoidance to control } \\
\text { Multiple Drones. }\end{array}$ & $\begin{array}{l}\text { A system control for more than } \\
\text { one drone which consists a } \\
\text { property of avoiding the collisions } \\
\text { autonomously. This system uses } \\
\text { detection device composed of } \\
\text { embedded control device and } \\
\text { ultrasonic sensors. The author has } \\
\text { also designed a collision } \\
\text { avoidance algorithm and claims } \\
\text { that the work accuracy of the } \\
\text { whole system is more that } 90 \% \\
\text { [2]. }\end{array}$ \\
\hline 2. & 2016 & C.M.Eaton & $\begin{array}{l}\text { Unmanned Aerial System Control } \\
\text { for multiple scenario systems. }\end{array}$ & $\begin{array}{l}\text { Provides a system architecture } \\
\text { which can be modified depending } \\
\text { upon the requirements of the } \\
\text { mission and the platform. The } \\
\text { paper also discusses about } \\
\text { employment of UAS capabilities } \\
\text { and current research [3]. }\end{array}$ \\
\hline 3. & 2015 & Ogan, R. T. & $\begin{array}{l}\text { Applications and challenges of } \\
\text { Unmanned Aircraft Systems. }\end{array}$ & $\begin{array}{l}\text { Discusses about the issues present } \\
\text { in the autonomous intelligent } \\
\text { control of UAVs and the four- } \\
\text { layer hierarchal control } \\
\text { architecture [8]. }\end{array}$ \\
\hline 4. & 2015 & Sahingoz, O. K. & $\begin{array}{l}\text { Bezier curve-based flyable } \\
\text { trajectories are generated for } \\
\text { multi-UAV systems using the } \\
\text { algorithm of parallel genetic. }\end{array}$ & $\begin{array}{l}\text { Discusses about the Genetic } \\
\text { Algorithm (GA) used to construct } \\
\text { flyable trajectory for multiple } \\
\text { UAV system. The purposed } \\
\text { system is written in the Java } \\
\text { language with a GUI for } \\
\text { displaying the results [9]. }\end{array}$ \\
\hline 5. & 2015 & $\begin{array}{l}\text { Turker, T., Sahingoz, O. K., } \\
\& \text { Yilmaz, G }\end{array}$ & $\begin{array}{l}\text { Application in radar threatening } \\
\text { environment using simulated } \\
\text { annealing algorithm for } 2 \mathrm{~d} \text { path } \\
\text { planning for UAS. }\end{array}$ & $\begin{array}{l}\text { Explains Simulated Annealing } \\
\text { (SA) Algorithm used for } \\
\text { optimizing the 2D path for UAVs. } \\
\text { The paper discusses about the } \\
\text { easy threat avoidance methods } \\
\text { and applies it to results using SA } \\
\text { to avoid regular circular radar } \\
\text { hazards [10]. }\end{array}$ \\
\hline 6. & 2013 & $\begin{array}{l}\text { Mao, Z., Zhang, Zheng, Z } \\
\text { Liu, W., Liu, J. }\end{array}$ & $\begin{array}{l}\text { For path planning of UAVs by } \\
\text { using the Cooperative and } \\
\text { geometric learning algorithms }\end{array}$ & $\begin{array}{l}\text { The author proposed an algorithm } \\
\text { known as Cooperative and } \\
\text { Geometric Learning (CGL) to } \\
\text { determine and clear up the issues } \\
\text { occurring in planning path for } \\
\text { UAVs. The authors also discusses } \\
\text { about the contribution of CGL } \\
\text { [11]. }\end{array}$ \\
\hline
\end{tabular}




\section{CLASSIFICATION OF UAVs}

There are mainly four UAV categories in the market. All these three categories have different technology implemented in them.

\subsection{Mini UAVs}

The mini UAVs are designed to work and fly below the altitude of 300 . The mini UAVs weighs below 30 kilograms and has the ability to fly from 150 to 300 meters. The mini UAVs can have flight duration of 3.4 hours with cruise speed 60-100 kilometers per hour. It can consists of various features such as mapping functions, secure digital communication, Video functions, automatic way point navigation and much more. They are designed in such a way that they can be used by the civil as well as for the commercial purpose also.

\subsection{Micro UAVs}

The micro UAVs also designed to fly at a low altitude. The micro UAVs weighs below 10 kilograms and can have a cruise speed of $45-55 \mathrm{~km} / \mathrm{h} / 28-34 \mathrm{mph}$. The micro UAVS can be provisioned and transported easily because of its small size and low weight. The designing of these UAVs has been done keeping in mind about the commercial and civil applications.

\subsection{Tactical UAVs}

The tactical UAVs have the capability to fly between an altitudes ranging from 3,000 to 8,000 meters. They can have a weigh from $150 \mathrm{~kg}$ to $1,500 \mathrm{~kg}$. The only challenge with these UAVs that they require a pleasantly free from wind environmentfor safe takeoffs and landings. It supports feature of route resonance, proving detailed terrain information and determine possible attack zones. They can be used in military fields as the have the capability to find a pinpoint target. A tactical UAV with Autonomous Takeoff and Landing System (ATOLS) can not only reduce the cost of training, manning but also minimizes the operational burdens.

\subsection{Strategic UAVs}

The strategic UAVs are the heaviest UAVs. These UAVs can travel up to highest altitude and weighs greatest among others. The Strategic UAVs are capable of transporting heavy and delicate payloads. They have the ability to travel a long distance for a long time and over a great altitude of about 20,000 meters or more. They can be used in military for advanced surveillance and monitoring atmosphere. Because of long travelling capability these UAVs can be beneficial for the commercial use also.

\section{SYSTEM REQUIREMENTS}

In this modern world, the demands to have UAV as Reconnaissance, Intelligence, Target Acquisition Systems and Surveillance are increasing exponentially. The condition of UAVs mainly depends on the missions, operations, tasks that need to be done. Some of the conditions that system should follow are Cost-effective, reliable, maintainable, useful and operationally available.

\subsection{System Reliability}

System Reliability is a very important parameter which is a direct result of the system hardware and system maturity. Software Bugs and Hardware failures are common during development stage of the system thus affecting the system reliability. Among UAV systems, engine and software are seen to be the most complex and critical parts. Reliability is very critical for the major and most expensive UAV systems that have the ability to carry multiple payloads for extended duration.

\subsection{Maintainability}

It is the ability of the UAVs to be sustained to the working condition when the sustainment is being executed by users having certain techniques usingthe prescribed methods and data at every step of repair and maintenance. The specifications that are used isDirect maintenance man-hours per flight hour (DMMH/FH) or Mean Time To Repair (MTTR), to calculate the system maintainability. The features of accessibility, Built-in-Test (BIT) and other automatic testing applicabilityimprovemaintainability.

\subsection{Operational Availability}

Operational Availability is the factor determining the chances that a system is functional and is ready to execute its desired task at any point of time. It is usually predicted on the basis of the design the reliable and maintainable factors of the system, and consider the respective maintenance methods (corrective and preventive) and supply (logistics).

\subsection{Sustainability}

Sustainability has the capability to maintain the necessary step and time period of the functional activity in order to accomplish military requirements. It is a parameter that provides and maintains steps of forces, material, and consumables that are important to support military efforts [12].The system should have the capability in order to complete a constant operations of specified period in the functional field without any guide from the users other than the system crew, in order to accomplish high availability rates. This is extremely necessary for the highly mobile diplomatic UAS.

\section{PATH PLANNING AND SAFETY CONTROLS}

The process of generating an automatic alternate path for an Unmanned Aerial Vehicle is known as Path Planning. It is complex problem which needs to be solved. It is important to optimize the paths to minimize the risks caused because of any attacker hazards, to reduce the fuel devouring acquired by diverging from the original path, and to amplify supervision over already defined end functions [24]. Therefore, there is a need have predefined criteria to select path from among possibilities. They solve the issues by confiding the system to plan only in a horizontal plane and taking height as a unchangeable value. There are many algorithms which are very helpful in achieving these goals. Some of them are:

\subsection{Parallel Genetic Algorithm}

This algorithm is used for constructing flyable trajectory for multi-UAVs system at a constant altitude and in a known environment. It is implemented in a multi-core environment in parallel manner and therefore increases the performance of the system [9]. The parallel genetic algorithm helps in creating a feasible and effective path for the drones. And then these path are converted into smoothed flyable path by using Bezier curves.

\subsection{Simulated Annealing Algorithm}

This algorithm is used for achieve an optimal 2-D path radar constrained environment. This algorithm can also be used to avoid regular circular radar threats [10]. It provides satisfactory answers and results with the ability of escaping from the local minima by using capital agreement rule and the 
threat that proposed avoidance approach used to the excellent found answers making the threat-free path easily.

\subsection{Cooperative and Geometric Learning}

This approach is used in solving the problem involved in planning the path for Unmanned Aerial Systems (UAS) such as collision escape, exploitation, and sharing of information. The contributions of CGL are threefold: 1) The ideal way in terms of length of the path and measurement of risk from a specified point to the destination point can be measured. 2) In this algorithm, the reward matrix $G$ is measured in actual-time and adjustably updated according to the arithmetic distance and UAS sharesinformation about the risk. 3) Algorithm gets a reward matrix $G$ that results to an easy and efficient approach for planning the path of more than one UAV systems [11]. However, with the increase in the demand of drones in the market, the drone accidents and crimes via them are also increasing rapidly.UAVs possess the necessity of intelligence to plan efficiently along a way to an intended destination while escaping the problems such as other aerial vehicle or attacker hazards. So, for achieving these goalsthe controlling system should consist of camera for pattern recognitions, collaborative drone control by multiple drones and emergent controls in uncontrollable situation [25].

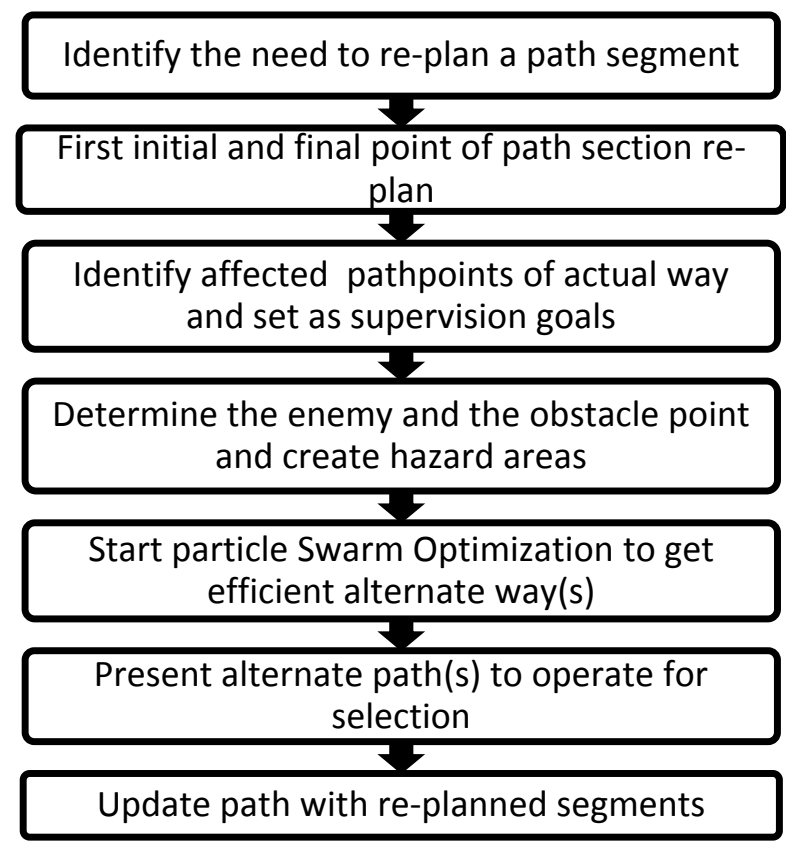

Fig. 1 Path Planning Process

\section{APPLICATIONS OF UAVs}

A lot of applications are utilized for remote sensing to which advantage is given because of the deployment of Unmanned Aerial Conveyance. In many scenarios, it is because of the cost involved in the missions, the requisite for more expeditious replication or the fact that observations that need to be executed in such an environment that may be hazardous to the crew.

The example that comes to our mind is the agreement of applications regarding remote sensing in the area of Excavation [23]. The important work is the recording and documentation of excavation areas, and to provide a 'bigger and broader view'. Essentiality for precision is not too big, albeit it's been proven that the example; height precision utilizing an aerial vehicle and results height models that can be compared to calculation of the ground laser scanner algorithms.

Herbage Auditing has withal been performed prosperously utilizing UAV systems. A Pathfinder Plus known as HALE Aerial vehicle, was acclimated to mark this on a coffee hacienda in Hawaii [14] other researcher have withal examined grasslands [15] andthese systems in Japan have been deliberated to be an necessary portionof farm accomplishments [16]. Expeditious replication imaging utilizing UAVs has been determined and has become remarkable. This has been established for road contingency paralleling [17] and for most of the cases of forest fire monitoring [18, 19, 20]. UAV systems have withal been purposed as stand to audit volcanoes [21]. An instance of the adaptability of Aerial Vehicles is their utilization in traffic auditing [22].

\section{PROBLEMS IN UAVS}

Some of the problems that are faced by the personnel that use UAVs are: The drones in today's time have limited payload capacity and flight endurance. After the fight of 15 to 30 minutes these drones are required to recharge the batteries again. While there are also such drones available which have the capability to carry payload up to twenty pounds. To make the situation more complex, therelationship between payload weight and flight endurance is inverse which signifies that the increase in load can lower the time of fight. The second difficulty found in UAVs is when the Competing solutions refuse to die, the large organizations does not want to alter the ways of handling the tasks that are mission critical without any test, plan and study. Some of the UAVs including drones may become an exceptional result, the traditional method of executing tasks have been tested from time to time. Third and major problem of using drones is about the issue of privacy, safety and security among the public. They public is clearly understand these issues related to the use of drones. None of us wants drones peeping into our windows. The public may agree to the law enforcement techniques of tracking down fleeing suspectsusing the drones,also they worry about the agencies of government that can usethese drones to spy on innocent citizens. And in today's world, everyone has a fear of the drones being used by the terrorists to guard out their goals and transfer explosives.

The other key safety areas are: The inability to agonize and evade other aerial vehicles in homogeneous way to manned airborne objects. There are susceptibilities in the direction and arrangement of UAV functions (example hacking, GPSjamming and cyber attacks). A deficiency of methodological and functional standards required to guide safe and consistent accomplishment of UAS. A deficiency of regime rules that are indispensable to securely forward the expedited integration of UAS into the national airspace system.

\section{IMPROVEMENT AREAS OF UAS}

The utilization of UASs is growing perpetually. In order to address future needs and authoritatively mandates for UAS, there is desideratum of a system which will have the capability to acclimate and can alter itself according to the incipient and transmuting requisites. Consequently, a plethora of research work has been done in order to build approaches that can enable path orchestrating for more than one mission. These algorithms withal include integration of the system capabilities and performance in order for amending identically tantamount. The control development of UAVs includes defines the capabilities and accomplishment of the conveyance that will lead to the amelioration the utility of the 
approaches in the authentic systems. Moreover, the integration in the system's performance, capabilities and the intricacies in these algorithms will yield an easy consideration of the functionalities and charge of the various approaches in authentic implementations.

As the time lines and the cost of development can involve difficulties such as the faculty to integrate many functional tasks into a system as they can engender a challenging market. For enabling magnification of UASs there is the desideratum to develop certain system design and approaches to recite for missions in multiple-scenario reducing the delay and costs. These amalgamations of the main system safety requisites, especially in the field of collision avoidance as well as boundary control, will avail establish that these systems are for fended, secure and trustworthy to operate.

\section{CONCLUSION}

In this paper, we have discussed various system requirements for making the Unmanned Aerial Vehicles more reliable and efficient in use that mainly depend upon the missions, operations, tasks that needs to be carried out, expectations are generally similar to each type. We have also discussed about the path planning algorithms such as Parallel Genetic Algorithm, Simulated Annealing Algorithm and Cooperative and Geometric Learning. These path planning algorithms are necessary for path optimization and to minimize fuel consumption, enemy threats, and to maximize reconnaissance over predefined targets. We have discussed various UAVs applications in Archaeology, Vegetation Monitoring, Rapid response imaging and traffic monitoring. Some various problems associated with UAVs are limited flight endurance and payload capacity, large corporations do not want to change the way they handle mission-critical tasks and of using drones is about the concerns among the public about privacy, security, and safety. As the demand of drones is increasing in the market there is a need to make the system adaptable and which can alter with new changes. This goal can be achieved by developing new and efficient algorithms.

\section{REFERENCES}

[1] N. A. S. Museum. Military Unmanned Aerial Vehicle, 2012.

[2] K.Surakul. A Control of Multiple Drones for Automatic Collision Avoidance

[3] C.M.Eaton.Multiple-Scenario Unmanned Aerial System Control: A Systems Engineering Approach and Review of Existing Control Methods, 2016

[4] Otto, R.P. Air Force ISR 2023: Delivering Decision Advantage; Headquarters United States Air Force: Washington, DC, USA, 2013.

[5] Dempsey, M.E. Intelligence, Surveillance, and Reconnaissance Joint Force 2020 White Paper; U.S. Army: Washington, DC, USA, 2014.

[6] Seffers, G. Joint Aerial Layer Network Vision Moves Toward Reality. Available online: http://www.afcea.org/content/?q=node/11123 (accessed on 19 August 2015).

[7] Valvanis, K.; Vachtsevanos, G. Future of Unmanned Aviation. In Handbook of Unmanned Aerial Vehicles; Springer: Dordrecht, The Netherlands, 2015; pp. 29933009.
[8] Ogan, R. T. (2015, April). Educating the next generation engineers for Unmanned Aircraft Systems applications and challenges. In SoutheastCon 2015 (pp. 1-7). IEEE.

[9] Sahingoz, O. K. (2014). Generation of bezier curvebased flyable trajectories for multi-UAV systems with parallel genetic algorithm. Journal of Intelligent \& Robotic Systems, 74(1-2), 499-511.

[10] Turker, T., Sahingoz, O. K., \&Yilmaz, G. (2015, June). $2 \mathrm{~d}$ path planning for uavs in radar threatening environment using simulated annealing algorithm. In Unmanned Aircraft Systems (ICUAS), 2015 International Conference on (pp. 56-61). IEEE.

[11] Zhang, B., Mao, Z., Liu, W., Liu, J., \&Zheng, Z. (2013, May). Cooperative and geometric learning for path planning of UAVs. In Unmanned Aircraft Systems (ICUAS), 2013 International Conference on (pp. 69-78). IEEE.

[12] DOD, Dictionary of Military and Associated Terms, March 1994

[13] E .Torun. UAV Requirements and Design Consideration, 2000

[14] Herwitz, S.R. et al.,2004. Imaging from an unmanned aerial vehicle: agricultural surveillance and decision support. Computers and Electronics in Agriculture 44 (2004) 49-61.

[15] Rango, A. et al., 2006. Using unmanned vehicles for rangelands: Current applications and future potentials. Environmental Practise, 8 (3), 159-169.

[16] Newcombe, L., 2007. Green fingered UAVs. Unmanned Vehicle, November 2007.

[17] Haarbrink, RB.,Koers, E., 2006. Helicopter UAV for Photogrammetry and Rapid Response. In: The International Archives of Photogrammetry, Remote Sensing and Spatial Information Sciences, Antwerp, Belgium, Vol. XXXVI-1/W44.

[18] Réstas, A., 2006. The regulation Unmanned Aerial Vehicle of the Szendro Fire Department supporting fighting against forest fires 1st in the world! Forest Ecology and Management, 234S (2006) S233

[19] Martínez-de Dios, J.R., et al., 2006. Experimental results of automatic fire detection and monitoring with UAVs. Forest Ecology and Management 234S (2006) S232.

[20] Casbeer, D.W. et al, 2006. Cooperative forest fire surveillance using a team of small unmanned air vehicles. International Journal of Systems Science 37 (6), 351-360.

[21] Buongiorno, M.F., 2005. Remote Sensing Application to Monitor Active Volcanoes. Use-HAAS Workshop \#1, Brussels, Belgium.(see http://www.usehaas.org/, accessed 6 May 2008)

[22] Puri, A. et al., 2007. Statistical Profile Generation for Traffic Monitoring Using Real-time UAV based Video Data. Proceedings of Mediterranean Conference on Control \& Automation 2007, Athens, Greece.,

[23] Çabuk, A., Deveci A. \&Ergincan F. 2007. Improving Heritage Documentation. GIM International 21, 9, September 2007. 
[24] L.D Swartzentruber, Iowa State University: improving path planning of unmanned aerial vehicles in an immersive environment using metapaths and terrain information, 2009.
[25] Okutake, T., Uchida, N., \& Yamamoto, N. (2016, July). A Collaborative Safety Flight Control System for Multiple Drones. In Innovative Mobile and Internet Services in Ubiquitous Computing (IMIS), 2016 10th International Conference on (pp. 371-375), IEEE.

[26] 